\title{
Effects of viscosity on droplet formation and mixing in microfluidic channels
}

\author{
Joshua D. Tice, Adam D. Lyon, Rustem F. Ismagilov* \\ Department of Chemistry, The University of Chicago, 5735 S. Ellis Avenue, Chicago, IL 60637, USA
}

Received 4 August 2003; received in revised form 22 October 2003; accepted 7 November 2003

\begin{abstract}
This paper characterizes the conditions required to form nanoliter-sized droplets (plugs) of viscous aqueous reagents in flows of immiscible carrier fluid within microfluidic channels. For both non-viscous (viscosity of $2.0 \mathrm{mPa}$ ) and viscous (viscosity of $18 \mathrm{mPa}$ ) aqueous solutions, plugs formed reliably in a flow of water-immiscible carrier fluid for Capillary number less than 0.01, although plugs were able to form at higher Capillary numbers at lower ratios of the aqueous phase flow rate to the flow rate of the carrier fluid (in all the experiments performed, the Reynolds number was less than 1). The paper also shows that combining viscous and non-viscous reagents can enhance mixing in droplets moving through straight microchannels by providing a nearly ideal initial distribution of reagents within each droplet. The study should facilitate the use of this droplet-based microfluidic platform for investigation of protein crystallization, kinetics, and assays.
\end{abstract}

(C) 2003 Elsevier B.V. All rights reserved.

Keywords: Viscosity; Microfluidic; Mixing; Multiphase; Droplet; Plug

\section{Introduction}

This paper discusses the effects of viscosity on the formation of droplets and on mixing inside the droplets moving through straight microfluidic channels. The formation of droplets in two-phase flow in microchannels has attracted significant attention. Formation of patterns in the flows far from equilibrium [1] and the effects of wettability on the patterns generated in two-phase flows [2] have been described. Preparation of monodisperse emulsions [3] and the use of flow-focusing techniques to control both size and distribution of monodisperse and polydisperse emulsions [4] have been reported. Segmented flow has been incorporated into a DNA analyzer [5], and droplets have been used in microchannels to perform and enhance two-phase chemical reactions $[6,7]$. We have recently shown that it is possible to form droplets of multiple aqueous reagents in a flow of water-immiscible carrier fluid, transport the droplets through microchannels without dispersion, and mix the contents of the droplets by chaotic advection in winding channels [8]. We refer to droplets that contact all four walls of a microchannel but do not wet the walls as plugs. We use

\footnotetext{
* Corresponding author. Tel.: +1-773-702-5816; fax: +1-773-702-0805.

E-mail address: r-ismagilov@uchicago.edu (R.F. Ismagilov).
}

the word "contact" somewhat loosely, because even though plugs press against all four walls of the microchannel, they are prevented from intimate contact with the walls by a thin film of carrier fluid $[9,10]$. Previously, we have characterized the formation of these plugs and mixing within the plugs using fluids that had matched viscosities, all approximately equal to that of water $[11,12]$. Recently, we have begun to apply this plug-based microfluidic platform to the manipulation of viscous protein solutions for functional and structural studies. We used this platform to screen hundreds of protein crystallization conditions inside nanoliter-sized plugs, consuming less than $4 \mathrm{nl}$ of protein solution for each crystallization trial [13]. We also demonstrated that the platform can be used to study millisecond enzyme kinetics using only nanoliters of reagents [14]. Handling of viscous solutions (e.g. solutions of polyethylene glycol and concentrated protein and enzyme solutions) was an important issue in both of the studies. Consequently, it has become apparent that the effects of viscosity on the formation of plugs and on the mixing within plugs should be characterized.

Here we investigated the formation of plugs in multiphase flows where the fluids were more viscous than water. We also investigated the formation of plugs in flows where the viscosities of the aqueous and carrier fluids were different from one other. In both cases, the flows form plugs up to a certain threshold velocity, like flows of fluids of low, matched vis- 
(a)

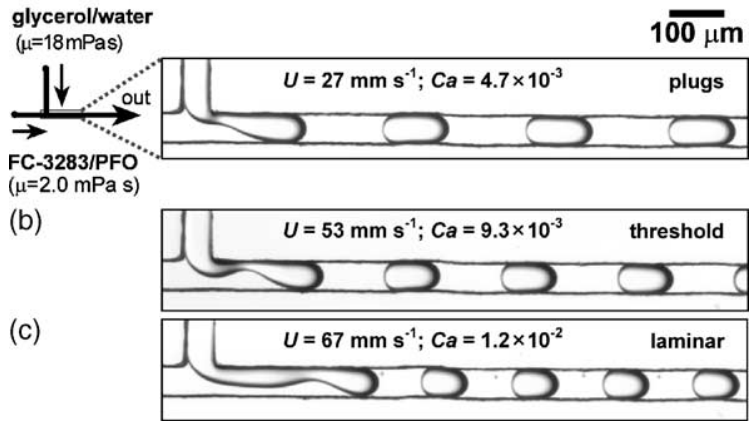

Fig. 1. Visualization of the three regimes of behavior observed in two-phase flows of viscous fluids. (a) Left: a scheme of the microfluidic network. Right: a microphotograph of formation of plugs at low value of $C a=4.7 \times 10^{-3}$. (b) A microphotograph of flow that is in transition to laminar flow. The flow velocity was $53 \mathrm{~mm} \mathrm{~s}^{-1}$, and the length of the laminar segment was $80 \mu \mathrm{m}$. The Capillary number, $C a=9.3 \times 10^{-3}$. (c) A microphotograph of flow where the length of the laminar segment is $200 \mu \mathrm{m}$. The flow velocity was $67 \mathrm{~mm} \mathrm{~s}^{-1}$, and $C a=1.2 \times 10^{-2}$. In all microphotographs, $w f=0.5$. The cross-sectional dimensions of the channels were $50 \mu \mathrm{m} \times 50 \mu \mathrm{m}$.

cosity. However, multiphase flow reached the threshold at lower flow velocities when viscous solutions were used. For all the experiments, the value of the Reynolds number was low $(R e<1)$, and we have previously shown that at these values, $R e$ has a negligible effect on formation of plugs and mixing inside them [11]. We quantify the threshold veloc- ities in terms of the Capillary number, $\mathrm{Ca}$, and water fraction, $w f$, showing that $C a$ can be used to reliably predict thresholds for combinations of fluids that have matched viscosities. $C a=U \mu / \gamma$, where $U\left(\mathrm{~m} \mathrm{~s}^{-1}\right)$ is the flow velocity, $\mu\left(\mathrm{kg} \mathrm{m}^{-1} \mathrm{~s}^{-1}\right)$ the dynamic viscosity, and $\gamma\left(\mathrm{kg} \mathrm{s}^{-2}\right)$ the surface tension at the interface between the aqueous phase and the carrier fluid. The Capillary number "represents a measure of viscous stresses relative to interfacial tension stresses" [15]. Water fraction, $w f$, is the ratio of the volumetric flow rate of the aqueous phase to the total volumetric flow rate of carrier fluid and aqueous phase. We also demonstrate that although mixing still proceeds by recirculating flows in plugs of viscous reagents, mixing is affected by the presence of viscous reagents. Although viscous reagents are expected to mix more slowly than non-viscous reagents, we show that for certain conditions, a combination of viscous and non-viscous reagents mix faster than a combination of all non-viscous reagents. The results of these investigations should be important for studies that utilize plug-based microfluidic platforms to manipulate proteins, biological fluids, and organic reagents [16-18].

\section{Experimental}

The experiments were performed as described previously [11], with the following additions: We measured
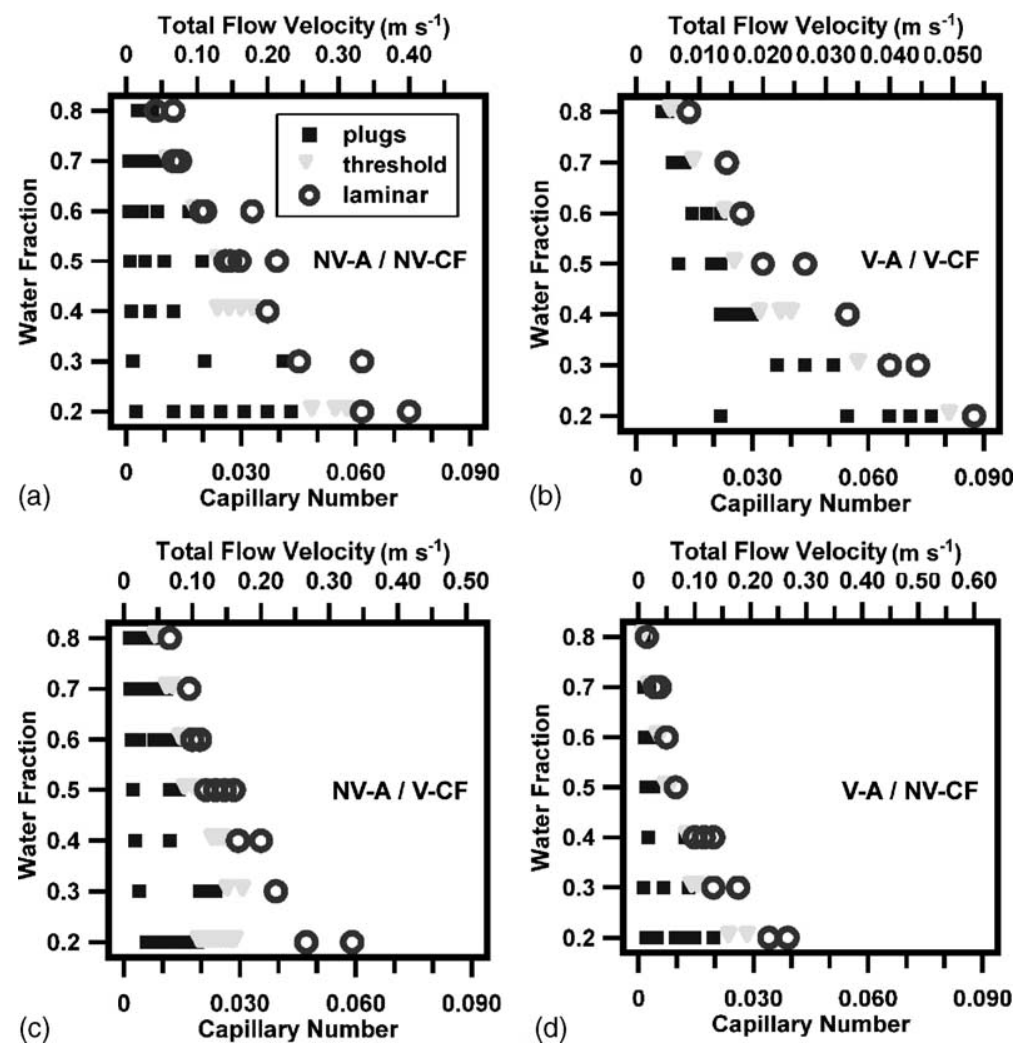

Fig. 2. Phase diagrams characterizing plug formation in four systems of viscous/non-viscous aqueous solutions and carrier fluids. The four combinations were: (a) NV-A and NV-CF, (b) V-A and V-CF, (c) NV-A and V-CF, (d) V-A and NV-CF. For (c) and (d), Ca was calculated using the viscosity of the non-viscous phase. Abbreviations are defined in Section 2. 


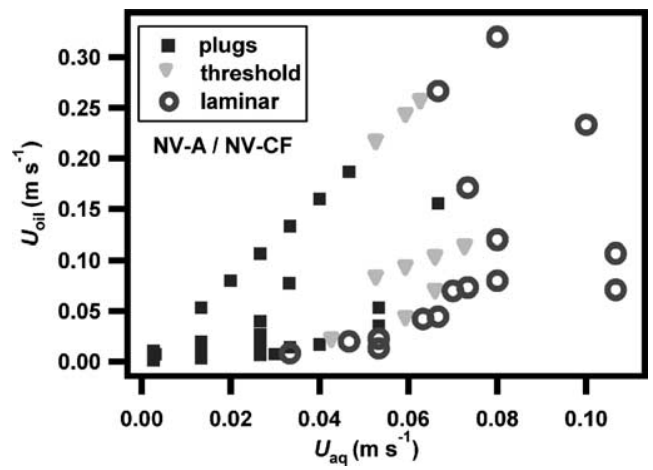

Fig. 3. A phase diagram characterizing plug formation in the system of NV-A and NV-CF. Points are plotted against the superficial flow velocities of the two fluids. These velocities were calculated just upstream of the junction where plugs formed.

the viscosity of fluids using a glass capillary viscometer (British-Standard, U-Tube; Cannon Instrument Company). In the experiments that characterize formation of plugs (reported in Figs. 1-3), non-viscous aqueous solution (NV-A) was a solution of $24 \%$ glycerol in water $(\mathrm{m} / \mathrm{m})$, $\mu=2.0 \mathrm{mPa}$. Viscous aqueous solution (V-A) was a solution of $68 \%$ glycerol in water $(\mathrm{m} / \mathrm{m}), \mu=18 \mathrm{mPas}$. Non-viscous carrier fluid (NV-CF) was a 10:1 (v/v) mixture of $3 \mathrm{M}$ fluorinated fluid $\mathrm{FC}-3283$ and $1 \mathrm{H}, 1 \mathrm{H}, 2 \mathrm{H}, 2 \mathrm{H}$ perfluoro-1-octanol (PFO), $\mu=2.0 \mathrm{mPa}$. Viscous carrier fluid $(\mathrm{V}-\mathrm{CF})$ was a 10:1 (v/v) mixture of perfluoroperhydrophenanthrene (PPP) and PFO, $\mu=18 \mathrm{mPas}$. The surface tensions between various aqueous solutions and carrier fluids (as determined by the pendant-drop method [19-21]) were as follows: $10.9 \pm 0.5 \mathrm{mN} \mathrm{m}^{-1}$ between $\mathrm{VA}$ and $\mathrm{V}-\mathrm{CF}, 13.4 \pm 0.5 \mathrm{mN} \mathrm{m}^{-1}$ between $\mathrm{V}$-A and NV-CF, $11.4 \pm 0.5 \mathrm{mN} \mathrm{m}^{-1}$ between NV-A and V-CF, and $10.6 \pm 0.5 \mathrm{mN} \mathrm{m}^{-1}$ between NV-A and NV-CF. In the experiments that characterize mixing in plugs (reported in Fig. 4), the dark colored streams were $0.07 \mathrm{M}$ solutions of $\mathrm{Fe}(\mathrm{SCN}) x^{(3-x)+}$ complexes. The colored solution in Fig. 4a was made with NV-A, and the viscosity of the solution was $2.0 \mathrm{mPa}$ s. The colored solution in Fig. $4 \mathrm{~b}$ was made with

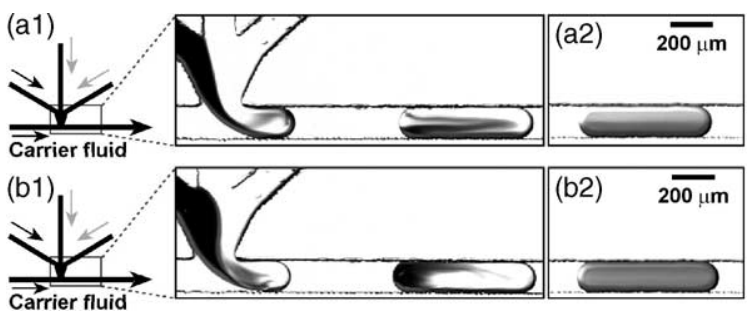

Fig. 4. Comparison of mixing behavior in plugs when (a) all three aqueous streams were non-viscous with (b), when one of the aqueous streams was viscous. (a1) and (b1) Left: a scheme of the microfluidic network. Right: microphotographs of plug formation. (a2 and b2) Microphotographs of plugs that had traveled identical distances of $\sim 14 \mathrm{~mm}$ for identical periods of time ( $\sim 2.1 \mathrm{~s})$ through the straight channel. Channels had cross-sectional dimensions of $150 \mu \mathrm{m} \times 100 \mu \mathrm{m}$. The solutions are described in Section 2. In (a) and (b), $U=6.7 \mathrm{~mm} \mathrm{~s}^{-1}, w f=0.5$, and the plugs had virtually identical volumes.
$\mathrm{V}-\mathrm{A}$, and the viscosity of the solution was $19 \mathrm{mPa}$. The colorless aqueous streams in Fig. 4 were a $0.2 \mathrm{M}$ solution of $\mathrm{KNO}_{3}$ in NV-A. The viscosity of the colorless solution was $2.1 \mathrm{mPa}$ s. Non-viscous carrier fluid (NV-CF) was used in both of the experiments reported in Fig. 4.

\section{Results and discussion}

\subsection{Forming plugs of solutions with viscosity higher than water}

We investigated the formation of plugs with four combinations of aqueous solution and carrier fluid and found that plugs could be formed reliably, regardless of the viscosity of the fluids. The combinations of fluids were (1) NV-A and $\mathrm{NV}-\mathrm{CF}$, (2) V-A and V-CF, (3) NV-A and V-CF, and (4) $\mathrm{V}-\mathrm{A}$ and NV-CF (abbreviations are defined in Section 2).

For all the combinations, the multiphase flow exhibited three regimes of behavior: (i) At low flow velocities $(7.0 \times$ $10^{-4} \leq \mathrm{Ca} \leq 7.6 \times 10^{-2}$ ), plugs immediately separated from the aqueous stream at the junction where the aqueous stream met the stream of carrier fluid (Fig. 1a). The length of plugs and the spatial period, or center-to-center distance between adjacent plugs, were reproducible. (ii) As the flow velocity and $\mathrm{Ca}$ were increased to a certain threshold $\left(1.9 \times 10^{-3} \leq C a \leq 8.2 \times 10^{-2}\right)$, plugs did not separate immediately at the junction. Instead, the aqueous stream formed a short (one to two times the width of the channel) laminar segment beyond the junction before plugs separated from the stream (Fig. 1b). (iii) At higher flow velocities and Ca $\left(2.2 \times 10^{-2} \leq C a \leq 1.1 \times 10^{-1}\right)$, the laminar segment propagated further downstream and its length fluctuated (Fig. 1c). The size of plugs also decreased noticeably. The three regimes may be explained very simplistically in terms of the Capillary number. The ratio $\gamma / \mu$ is an expression of the velocity at which the interface between the two phases may move under the force of surface tension, resisted by the viscosity of the fluids. As long as the flow velocity is much lower than the interfacial velocity $\gamma / \mu$, the surface tension dominates and the plugs snap off cleanly (regime (i)). As the velocity of the driving flow increases, the interfacial velocity is not high enough for clean snap-off, and fluids proceed laminar for some distance before the snap-off occurs.

We quantified the three regimes of flow for the four combinations of aqueous solution and carrier fluid in terms of $C a$ and $w f$. The surface tensions between aqueous solution and carrier fluid were similar for all four combinations of fluids; the only components of $\mathrm{Ca}$ varied in this study were $\mu$ and $U$. The results are displayed as phase diagrams in Fig. 2. For all four combinations of fluids, the regions of the phase diagrams that correspond to regime (i) have the same general shape. Threshold values of $C a$ were higher for lower $w f$. For two of the combinations of fluids, the viscosity of the aqueous solution matched the viscosity of the car- 
rier fluid. From the diagrams characterizing combinations of NV-A and NV-CF (Fig. 2a) and V-A and V-CF (Fig. 2b), it was clear that $C a$ was important for predicting the behavior of the flow. Viscosities of the fluids differed by nearly a factor of ten, and the flow velocities at a threshold for a given $w f$ were about ten times lower in the flow of viscous fluids. However, the threshold values of $\mathrm{Ca}$ for a given $w f$ were nearly identical between the two diagrams. For a given $w f$, the threshold values of $C a$ differed by a factor between 1.1 and 1.4, except for $w f=0.2$ where the threshold values of $\mathrm{Ca}$ differed by a factor of 1.7 .

For flows of two fluids with different viscosities, it was unclear which viscosity should have been used to calculate $\mathrm{Ca}$ to predict threshold flow velocities. In the phase diagrams constructed for two combinations of fluid where there was a contrast between the viscosity of the aqueous solution and that of the carrier fluid (Fig. 2c and d), the viscosity of the non-viscous phase was used to calculate $\mathrm{Ca}$. It appeared that $\mathrm{Ca}$, calculated this way, did not predict the behavior of the flow as accurately as in the combinations of fluids of matched viscosities. For example, at $w f=0.5$, threshold values of $C a$ differ by a factor of 1.5 between NV-A/V-CF and NV-A/NV-CF, and threshold values differ by a factor of 3.2 between $\mathrm{V}-\mathrm{A} / \mathrm{NV}-\mathrm{CF}$ and NV-A/NV-CF. It is interesting to consider that plugs of viscous solution may be formed at higher flow velocities using non-viscous carrier fluid rather than viscous carrier fluid.

Such phase diagrams have been often presented in the coordinates of velocities (or volumetric flow rates) of the two fluids $[2,4,7,22]$. We have replotted the data shown in Fig. 2a to underline the similarities of the phase diagram presented here and the ones observed previously (Fig. 3). We used the superficial flow velocities of each fluid, calculated by dividing the volumetric flow rate of the fluid by the cross-sectional area of the channel.

\subsection{Mixing in plugs of solutions with viscosity higher than water}

When two reagents flow laminar in microchannels, mixing is slow and proceeds by diffusion. The time needed to mix two reagents by diffusion is $t_{\text {diff }}=s t l^{2} / 2 D$ where $s t l(\mathrm{~m})$ is striation length, the distance that a reagent must diffuse to mix with another reagent, and $D\left(\mathrm{~m}^{2} \mathrm{~s}^{-1}\right)$ the diffusion coefficient. In a plug, the initial stl is on the order of the width of the plug (determined by the width of the channel). In long plugs formed at high water fractions, stl is approximately half of the width of the plug. The initial stl may be reduced further by the eddy present during the formation of shorter plugs formed at lower water fractions [11]. The value of $D$ is lower for viscous solutions and increases mixing time.

If reagents are localized within plugs, however, they can be mixed rapidly by re-circulating flows [11]. There are two vortices of flow in a plug moving through a straight microchannel, one in the upper half of the plug and another in the lower half (as viewed in Fig. 4) [23]. In principle, these vortices can enhance mixing by reducing stl. However, we have previously shown that the efficiency of mixing in straight channels depends significantly upon the initial distribution of reagents in each plug [11]. If one reagent is isolated in the upper half of the plug and another is isolated in the lower half, then mixing is not enhanced by internal flow. When reagents of matched viscosity formed long plugs at high $w f \sim 0.5$, this initial distribution was observed (Fig. 4a1).

For faster mixing by recirculation, one reagent should be initially distributed to the back of the plug, and the other reagent should be distributed to the front [11]. In this way, each reagent is divided equally between the two vortices. When one stream of $\mathrm{V}-\mathrm{A}$ and two streams of NV-A were simultaneously injected into a flow of NV-CF (V-A was injected through the leftmost inlet), the V-A initially collected in the back of the plug (Fig. 4b1). The distribution of the reagents, therefore, was nearly ideal, and for the conditions shown in Fig. 4, a viscous and a non-viscous reagent mixed faster than reagents of low, matched viscosities in long plugs (Fig. 4a2 and b2). (Our previous studies show that longer plugs form at higher water fractions [11].) For this combination of fluids, threshold values of $\mathrm{Ca}$ were identical to those found for a combination of $\mathrm{V}-\mathrm{A}$ and NV-CF. If $\mathrm{V}-\mathrm{A}$ was flowed through the rightmost inlet, then plugs formed only for significantly lower values of $\mathrm{Ca}$.

\section{Conclusion}

Plugs may be formed in multiphase flows of fluids with viscosities significantly higher than that of water. We have demonstrated that $\mathrm{Ca}$ can be used to predict the conditions necessary to reliably form plugs when the fluids in the flow have matched viscosities. In flows where there was a contrast between the viscosity of the aqueous solution and the viscosity of the carrier fluid, threshold values of $\mathrm{Ca}$ differed from the threshold values of $\mathrm{Ca}$ of flows of fluids with matched viscosities by a factor of 1.5-3.4. We also observed that two aqueous reagents of differing viscosities may mix more rapidly than two aqueous reagents with matched viscosities.

We conclude that this plug-based microfluidic platform is capable of accommodating viscous fluids, although several questions remain to be addressed. We used the viscosity of the non-viscous phase to calculate threshold values of $\mathrm{Ca}$ for flows of fluids with contrasting viscosities. In this case, threshold values of $\mathrm{Ca}$ are lower than those of a flow of fluids with matched viscosity. When we used the viscosity of the viscous phase, the threshold values were higher than those for a flow of fluids with matched viscosity. Therefore, it is important to establish how to average these viscosities to obtain threshold values of $\mathrm{Ca}$ that are consistent for all combinations of fluids. We have previously shown that mixing can be accelerated by increasing the velocity of the flow. Another issue to be investigated is whether geometries of 
microfluidic channels can be designed to increase the threshold values of $\mathrm{Ca}$ for a given combination of fluids to permit higher flow velocities. In the rectangular channels that we have been using, mixing in plugs relies on the recirculating flows caused by viscous stresses that act on the plug. When a plug of viscous fluid moves in the presence of a non-viscous carrier fluid, however, a thin film of carrier fluid with thickness $h(\mathrm{~m})$ lubricates the plug [9]. It has been shown for circular channels that $h \sim \mathrm{Ca}^{2 / 3}$ [24,25]. This relation predicts that at significantly high flow velocities, $h$ will be large, suggesting that most of the viscous stresses in a flow will be dissipated in the lubricating film. An important task will be to develop a mathematical model to determine the extent to which shear stresses are diminished by the lubricating film in channels with rectangular cross-sections. The lubrication makes mixing using chaotic advection in winding channels less effective, but also reduces the pressure gradient required to transport the viscous reagents through the microchannel. One would be able to use this model to balance these positive and negative effects of lubrication. Once these questions are addressed, this system will become especially useful for handling viscous fluids in microchannels in a predictable and controllable manner.

\section{Acknowledgements}

This work was supported by the Camille and Henry Dreyfus New Faculty Award, Chicago MRSEC funded by NSF, the ONR Young Investigator Award N00014-03-10482, the NIH (R01 EB001903), and by the Beckman Foundation. The work was performed at the Chicago MRSEC microfluidic facility. We thank H. Song and C.J. Gerdts for performing photolithography at MAL of UIC and for advice. We thank M.R. Bringer for helpful discussions.

\section{References}

[1] T. Thorsen, R.W. Roberts, F.H. Arnold, S.R. Quake, Phys. Rev. Lett. 86 (2001) 4163.

[2] R. Dreyfus, P. Tabeling, H. Willaime, Phys. Rev. Lett. 90 (2003).

[3] S. Sugiura, M. Nakajima, S. Iwamoto, M. Seki, Langmuir 17 (2001) 5562.

[4] S.L. Anna, N. Bontoux, H.A. Stone, Appl. Phys. Lett. 82 (2003) 364.

[5] M.A. Burns, B.N. Johnson, S.N. Brahmasandra, K. Handique, J.R. Webster, M. Krishnan, T.S. Sammarco, P.M. Man, D. Jones, D. Heldsinger, C.H. Mastrangelo, D.T. Burke, Science 282 (1998) 484.

[6] J.R. Burns, C. Ramshaw, Chem. Eng. Commun. 189 (2002) 1611.

[7] N. de Mas, A. Gunther, M.A. Schmidt, K.F. Jensen, Ind. Eng. Chem. Res. 42 (2003) 698.

[8] H. Song, J.D. Tice, R.F. Ismagilov, Angew. Chem. Int. Ed. 42 (2003) 768.

[9] J. Bico, D. Quéré, J. Fluid Mech. 467 (2002) 101.

[10] J. Bico, D. Quéré, J. Colloid Interface Sci. 247 (2002) 162.

[11] J.D. Tice, H. Song, A.D. Lyon, R.F. Ismagilov, Langmuir 19 (2003) 9127.

[12] H. Song, M.R. Bringer, J.D. Tice, C.J. Gerdts, R.F. Ismagilov, Appl. Phys. Lett. 83 (2003) 4664.

[13] B. Zheng, L.S. Roach, R.F. Ismagilov, J. Am. Chem. Soc. 125 (2003) 11170 .

[14] H. Song, R.F. Ismagilov, J. Am. Chem. Soc. 125 (2003) 14613.

[15] H.A. Stone, Annu. Rev. Fluid Mech. 26 (1994) 65.

[16] P.-A. Auroux, D. Iossifidis, D.R. Reyes, A. Manz, Anal. Chem. 74 (2002) 2623.

[17] P.-A. Auroux, D. Iossifidis, D.R. Reyes, A. Manz, Anal. Chem. 74 (2002) 2637.

[18] D.J. Beebe, G.A. Mensing, G.M. Walker, Annu. Rev. Biomed. Eng. 4 (2002) 261.

[19] F.K. Hansen, J. Colloid Interface Sci. 160 (1993) 209.

[20] F.K. Hansen, G. Rodsrud, J. Colloid Interface Sci. 141 (1991) 1.

[21] A.W. Neumann, J.K. Spelt, Applied Surface Thermodynamics, Marcel Dekker, New York, 1995.

[22] M.W. Losey, R.J. Jackman, S.L. Firebaugh, M.A. Schmidt, K.F. Jensen, J. Microelectromech. Syst. 11 (2002) 709.

[23] K. Handique, M.A. Burns, J. Micromech. Microeng. 11 (2001) 548.

[24] L.W. Schwartz, H.M. Princen, A.D. Kiss, J. Fluid Mech. 172 (1986) 259.

[25] F.P. Bretherton, J. Fluid Mech. 10 (1961) 166. 